\title{
Challenges in osteoporosis awareness and management: results from a survey of US postmenopausal women
}

\author{
E. Michael Lewiecki ${ }^{\mathrm{a}, \mathrm{b}}$, Deane Leader ${ }^{c}$, Richard Weiss ${ }^{c}$ and Setareh A. Williams ${ }^{c}$
}

${ }^{a}$ Department of Internal Medicine, University of New Mexico School of Medicine, Albuquerque, NM, USA; ${ }^{b}$ New Mexico Clinical Research \& Osteoporosis Center, Albuquerque, NM, USA; ' $R$ Radius Health, Inc, Waltham, MA, USA

\begin{abstract}
Background: Osteoporotic fractures are a serious public health burden, leading to excess morbidity, mortality, and high costs for post-fracture care.

Objectives: To evaluate the perception of osteoporotic fracture risk and to identify potential barriers associated with osteoporosis diagnosis and treatment in US postmenopausal women.

Methods: An online survey was conducted by The Harris Poll in 2017 that included 1,012 postmenopausal women 50 years and older. The survey comprised eight screening questions and 35 mandatory questions pertaining to lifestyle, general health, bone fracture awareness, osteoporosis, and demographics, with additional follow-up questions where applicable. An oversample of 102 women with osteoporosis and a fragility fracture (from a fall from a standing height or lower) yielded a total of 280 subjects with osteoporosis and a fracture.

Results: A total of 1,012 postmenopausal women were included in the survey; 501 self-reported a diagnosis of osteoporosis and 511 had no known osteoporosis diagnosis. Ninety-six percent of women with a first fracture but no osteoporosis diagnosis did not recall being told by their physicians that their fracture might be related to osteoporosis. Forty-four percent of respondents reported that they had not undergone screening for osteoporosis. Among those considered at risk for osteoporosis, $42 \%$ had not undergone screening. Only $45 \%$ of women surveyed agreed that a fracture is "a potential sign of more fractures/breaks to come". Twenty-four percent believed there is no way to build new bone at their age; $18 \%$ answered that they could not do much to reduce the risk of fracture at their age.

Conclusions: This survey reveals a lack of awareness of the diagnosis of osteoporosis and osteoporotic fracture risk among postmenopausal women. Future studies evaluating the perceptions of osteoporosis and its management are warranted.
\end{abstract}

\section{ARTICLE HISTORY}

Received 31 July 2018

Accepted 17 January 2019

\section{KEYWORDS}

Osteoporosis; fracture; treatment; treatment gap; crisis

\section{Introduction}

Osteoporosis is a serious yet under-recognized condition that is associated with significant adverse health and economic consequences ${ }^{1,2}$. Considering that the number of individuals 65 years and older will double in the US in $\sim 4$ decades (increasing from over 40 million in 2010 to more than 88 million by 2050), there is likely to be a substantial increase in the incidence of osteoporotic fractures and in the resultant socioeconomic burden. In the absence of meaningful action, as many as one in two Americans of 50 years and older (men and women) will have or be at high risk of osteoporosis by $2020^{2,3}$. Nationally, the projected increase in age-related osteoporotic fractures among both sexes is estimated to be more than $48 \%$, growing from 2.1 million in 2005 to more than 3 million in 2025. The direct costs from these fractures may exceed $\$ 25$ billion in 2025 , with the cumulative cost of incident fractures reaching $\$ 228$ billion for the 10-year period between 2016 and 2025 inclusive ${ }^{1}$.

Women comprise the group most affected by osteoporosis, with their age-adjusted incidence being $49 \%$ higher than that for men ${ }^{4}$. In 2015, more than 2 million fractures were documented in Medicare and commercial claims databases. Of these, women accounted for $\sim 70 \%$ of all fractures and represented $74 \%$ of the cost, with most fractures for women occurring between ages $75-84$ years ${ }^{5}$. The Healthcare Effectiveness Data and Information Set (HEDIS) is a tool used by more than $90 \%$ of America's health plans to measure performance on important dimensions of care and service for a number of disease areas ${ }^{6}$. In the case of osteoporosis, HEDIS assesses the number of women aged 65-85 years who suffered a fracture and who had either a bone mineral density

CONTACT E. Michael Lewiecki mlewiecki@gmail.com №w Mexico Clinical Research \& Osteoporosis Center, 300 Oak St NE, Albuquerque, NM 87106, USA. Color versions of one or more of the figures in the article can be found online at www.tandfonline.com/ijda.

$(4$ Supplemental data for this article is available online at https://doi.org/10.1080/21556660.2019.1579728.

(C) 2019 The Author(s). Published by Informa UK Limited, trading as Taylor \& Francis Group.

This is an Open Access article distributed under the terms of the Creative Commons Attribution-NonCommercial License (http://creativecommons.org/licenses/by-nc/4.0/), which permits unrestricted non-commercial use, distribution, and reproduction in any medium, provided the original work is properly cited.

www.tandfonline.com/ijda 
(BMD) test or prescription for a drug to treat osteoporosis in the 6 months after their fracture. The intent is to reduce the risk of fractures resulting from osteoporosis in older women ${ }^{7}$. A US observational study of women experiencing a first hip fracture between 2008 and 2013 showed that only $17 \%$ and $23 \%$ had evidence of osteoporosis assessment and/or treatment within 6 or 12 months of their fractures, respectively ${ }^{8}$. Results from a recently published survey of untreated postmenopausal women with osteoporosis and their physicians reported that patients themselves decided against pharmacological treatment in at least half of the cases of non-treatment. The most frequent reasons for this patient decision were concerns regarding side-effects, alternative non-prescription options (including behavioral modification), and questioning medication benefits ${ }^{9}$. It is, therefore, important to further identify and address factors that may contribute to the under-diagnosis and under-treatment of women who are at high risk for fracture.

The objective of this survey was to evaluate perceptions of osteoporotic fracture risk in a population of US postmenopausal women with and without a self-reported diagnosis of osteoporosis and to identify potential gaps in diagnosis and management of this condition.

\section{Methods}

An online survey was conducted by The Harris Poll from March 31 to April 17, 2017. Respondents were postmenopausal women 50 years and older living in the US. All respondents (not just those who met the qualifying criteria) were weighted to separate targets from the US Census Bureau's Current Population Survey (CPS) for two populations: women aged 50-64 years and women 65 years and older. All percentages were calculated to reflect weighted data. Data with a base less than 50 (i.e. 50 women or fewer in the original sample) were not reported, whereas data with a base greater than 50 respondents but fewer than 100 were deemed directional in nature. The variables used for weighting included age, education, race/ethnicity, region, household income, household size, marital status, employment status, and a propensity score, a proprietary Harris methodology that allows for a reduction in the bias inherent in selfselected online panels.

Survey respondents provided informed consent and were assured of complete confidentiality. Although subjects were not paid for completing the online survey, they received $\mathrm{HI}$ points (Harris Poll Online Rewards program). The survey was approved by IntegReview IRB, an accredited Institutional Review Board based in Austin, TX, and was compliant with the Health Insurance Portability and Accountability Act of 1996.

\section{Survey questions}

Subjects were asked to participate in a research survey regarding general health concerns for women 50 years and older (Supplementary Appendix). The survey comprised eight screening questions to determine eligibility. Those qualifying for inclusion were then asked 35 mandatory ("ask all") questions pertaining to lifestyle (10), general health (3), bone fracture awareness (3), osteoporosis (4), and demographics (15). Additional questions in each of these categories were asked if a response to an "ask all" question needed follow-up. For example, if a woman answered "Yes" to this survey question-"Have you discussed osteoporosis with your healthcare provider?"-she was then asked a follow-up question ("Who initiated the conversation you had with your healthcare provider about osteoporosis?") to which four responses were offered ("I did", "My healthcare provider", "Caregiver/family member/friend", and "I do not recall"). Those who answered "No" to any of the 35 mandatory "ask all" survey questions were not asked the follow-up question. Completion time for the survey was estimated to be 20 minutes.

\section{Subject classification}

A postmenopausal subject was classified as having osteoporosis if she self-reported an osteoporosis diagnosis by a healthcare professional. She was judged to be "at risk" for osteoporosis if she replied affirmatively to any of the following known risk factors for osteoporosis: (a) currently smokes cigarettes or smoked regularly within the past 5 years; (b) consumes 3 or more units of alcohol per day ( 1 unit is defined as one standard glass of beer [approximately $10 \mathrm{oz}$.]; one shot of whiskey [1 oz.]; a medium glass of wine [approximately 4 oz.]); (c) had a postmenopausal fracture; (d) father, mother, or sister has/had osteoporosis; (e) has confirmed rheumatoid arthritis; (f) currently uses or once used glucocorticoids for more than 3 months at a dose of $5 \mathrm{mg} /$ day or more of prednisolone (or equivalent doses of other glucocorticoids); and (g) experienced premature menopause (i.e. menopause occurring before age 45 years). The subsequent use of the term "fracture" relates to a fragility fracture, which was defined as having a fractured/broken bone resulting from a fall from a standing height or lower ${ }^{9,10}$.

\section{Statistical analysis}

To facilitate comparative analyses, the survey population of postmenopausal women was divided into one main group (all subjects $[A]$ ) and eight subgroups: women with (B) or without (C) osteoporosis; women without osteoporosis who were either at risk for the condition (D) or not at risk (E); women who had ever experienced a fracture and either had osteoporosis (F) or did not have osteoporosis (G); and women with osteoporosis who were aged 50-64 years $(\mathrm{H})$ or 65 years and older (I). An oversample of 102 women with osteoporosis and a fracture was identified to augment women from the main sample who met the same criteria, yielding a total of 280 subjects with osteoporosis and a fracture. The oversample was drawn from a sample of women over the age of 50 living in the US who had indicated they had been diagnosed with osteoporosis. They were then screened to confirm those criteria along with additional criteria of having a fragility fracture. Respondents from the 
Table 1. Survey respondent characteristics: 1,012 postmenopausal women aged at least 50 years.

\begin{tabular}{lcc}
\hline & $\begin{array}{c}\text { Osteoporosis } \\
\text { diagnosis }(n=501)^{\mathrm{a}}\end{array}$ & $\begin{array}{c}\text { No osteoporosis } \\
\text { diagnosis }(n=511)^{\mathrm{b}}\end{array}$ \\
\hline Mean age, years & 70 & 64 \\
Aged $\geq 65$ years, \% & 69 & 45 \\
Reported previous fracture, \% & 35 & 18 \\
Comorbidities, \% & & \\
Hypertension & 38 & 32 \\
Thyroid disease & 25 & 16 \\
Type 2 diabetes & 13 & 12 \\
Respiratory disease & 19 & 6 \\
Breast cancer & 12 & 4 \\
Rheumatoid arthritis & 10 & 3 \\
Heart attack & 4 & 2 \\
Stroke & 6 & 1 \\
Other cancers, excluding breast & 5 & 4 \\
\hline
\end{tabular}

${ }^{a}$ Does not include oversample.

${ }^{\mathrm{b}} 64 \%$ were at risk for osteoporosis based on the presence of multiple risk factors.

oversample were included only when discussing osteoporosis and a fracture. Statistical comparisons were conducted for $B$ vs $C, D$ vs $E, F$ vs $G$, and $H$ vs $I$. All data were analyzed in the aggregate. In addition, $t$-tests were conducted at the $95 \%$ confidence level $(\mathrm{CL})$, therefore representing a $p$-value $<0.05$.

\section{Results}

A total of 1,012 postmenopausal women were included in the survey; $49.5 \%$ (unweighted; $n=501$ ) self-reported a diagnosis of osteoporosis, and $50.5 \%$ (unweighted; $n=511$ ) had no known osteoporosis diagnosis. Among women with an osteoporosis diagnosis, $69 \%$ were 65 years and older, and $35 \%$ had a prior fracture. The survey also included an oversample of 102 women with osteoporosis and a fracture. These respondents were combined with the women from the main sample who met the same criteria, for a total of 280 women with osteoporosis and a fracture. The most common self-reported comorbidities were hypertension $(38 \%$ among women with an osteoporosis diagnosis and $32 \%$ among women without), thyroid disease (25\% and $16 \%)$, type 2 diabetes (13\% and $12 \%)$, and respiratory disease (19\% and 6\%) (Table 1). Based on the self-reported risk factors, the majority of survey respondents (64\%) who had no osteoporosis diagnosis were at risk for osteoporosis.

\section{Fractures and osteoporosis}

Postmenopausal women with osteoporosis and a fracture who were informed by their healthcare provider (HCP) that their fracture could be related to osteoporosis $(n=115)$ were asked what they thought caused their fracture prior to an HCP implicating osteoporosis. Of these, 35\% answered that they had no idea that osteoporosis was to blame until told by their HCP. Thirty-four per cent of these women thought their fracture might be due to osteoporosis, but were unsure, while only $31 \%$ of women with an osteoporosis diagnosis felt sure their fracture was due to osteoporosis.

According to the women surveyed who had discussed osteoporosis with their $\mathrm{HCP}, 58 \%$ of conversations with their

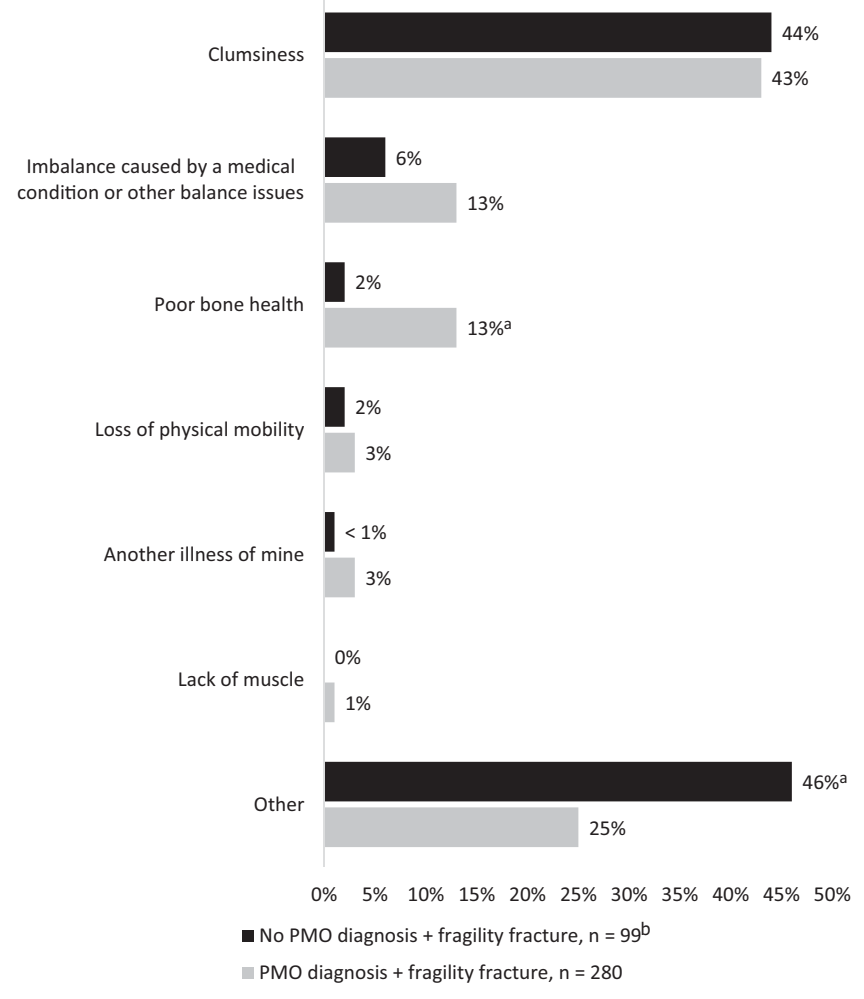

Figure 1. Respondents' perceived cause of most recent fracture. ${ }^{a} p<0.05 ; t-$ tests were conducted at the $95 \%$ confidence level $(\mathrm{CL})$, therefore representing a $p$-value $<0.05$. ${ }^{\mathrm{b}}$ Owing to sample size $<100$, these results are directional in nature.

HCPs about osteoporosis were initiated by the HCP, regardless of their risk status. HCP-initiated conversations were reported by $61 \%$ of women at risk for osteoporosis and by $49 \% *$ who were not at risk. However, $41 \%$ of women at risk for osteoporosis whose HCP brought up osteoporosis answered that the conversation with their HCP was started because of their age.

\section{Fractures as a sentinel event}

Only $13 \%$ of those with osteoporosis and $2 \% *$ of those without osteoporosis recognized that poor bone health may have caused their most recent fall-related fracture $(p<0.05)$. Forty-six percent of women with a fracture $(43 \%$ with osteoporosis and 44\%* without) blamed their most recent fracture on clumsiness rather than poor bone health. A further $13 \%$ of those without an osteoporosis diagnosis and $6 \%$ of those with a diagnosis attributed their fracture to balance problems brought on by medical conditions or other issues (Figure 1). More than a quarter of women $(29 \%$ with an osteoporosis diagnosis and $25 \%$ without) only associated fractures involving the hip and back with osteoporosis.

Many undiagnosed women with, or at risk for, osteoporosis were not aware of the risk factors for this condition. The most widely recognized risk factors among the respondents were a positive family history ( $76 \%$ not at risk, $78 \%$ at risk), cigarette smoking (56\% for both groups), premature

*Caution: Owing to the small sample size $(<100)$, these results are directional in nature. 


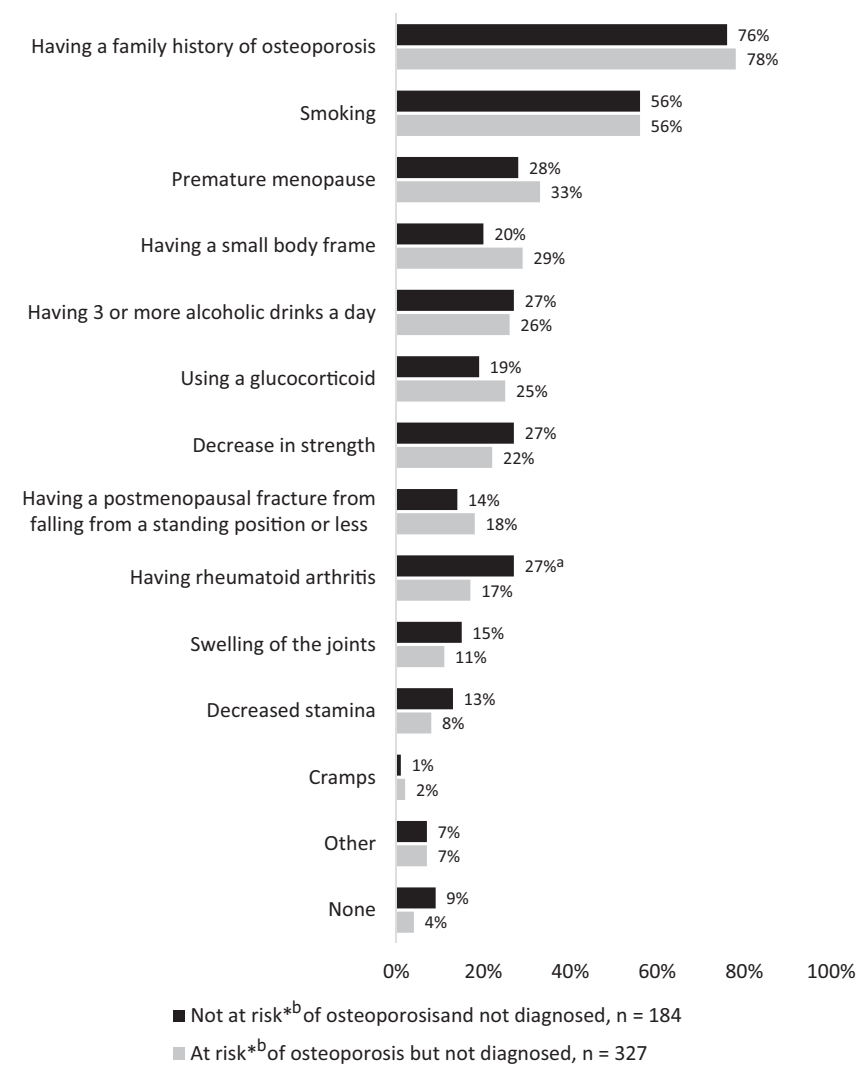

Figure 2. Awareness of risk factors for osteoporosis by postmenopausal women. ${ }^{a} p<0.05$. $t$-tests were conducted at the $95 \%$ confidence level $(C L)$, therefore representing a $p$-value $<0.05$. ${ }^{b}$ No current osteoporosis and at least one of the following: menopausal before age 45 years; rheumatoid arthritis; prior fracture; breast cancer; current smoker; $>3$ alcoholic drinks/day, has taken a glucocorticoid for $>3$ months at a time; mother, father, or sister has osteoporosis.

menopause (28\% and 33\%), having a small body frame $(20 \%$ and $29 \%)$, consuming 3 or more units of alcohol a day $(27 \%$ and $26 \%$ ), use of glucocorticoid agents (19\% and 25\%), and decreased strength (27\% and $22 \%)$. Forty-five percent of women agreed that a fracture potentially signifies future fractures, with undiagnosed women being less likely than diagnosed women to agree (43\% vs $57 \%$, respectively). However, only $18 \%$ of women at risk for osteoporosis and $14 \%$ not at risk identified having had a postmenopausal fracture as a risk factor for future fracture (Figure 2). A total of $31 \%$ of women with an osteoporosis diagnosis and $27 \%$ without believed that drinking milk or taking calcium supplements alone would reduce fracture risk. Additionally, $24 \%$ of postmenopausal women believed that there is no way to build new bone at their age, while $21 \%$ stated that the risk of a fracture cannot be reduced at their age (Figure 3 ).

\section{Management of fractures}

Among the 369 survey respondents who had experienced a recent fracture (272 with an osteoporosis diagnosis, 97 without an osteoporosis diagnosis), 31\% and 33\%* of women with and without an osteoporosis diagnosis who saw an HCP did not recall having a follow-up referral following their fracture event. Among those who reported that an HCP diagnosed them with their most recent fracture, 34\% who had an osteoporosis diagnosis and 43\%* without an osteoporosis diagnosis were referred to an orthopedic surgeon, $17 \%$ vs $13 \%$ to a primary care physician/geriatrician, $13 \%$ vs $8 \%$ to a physical therapist, $1 \%$ vs $2 \%$ to a rheumatologist, and less than $1 \%$ vs $0 \%$ to an endocrinologist (Figure 4). The majority of respondents did not recall being told that their fracture could possibly be related to osteoporosis. This was more common among women with a fracture and no established osteoporosis diagnosis than among those with fracture and an osteoporosis diagnosis (96\%* vs $61 \% *)$.

Respondents without an osteoporosis diagnosis were asked, 'Have you been screened for osteoporosis?' A total of $44 \%$ of respondents reported that they had not undergone screening for osteoporosis: $42 \%$ of those at risk for osteoporosis, $48 \%$ not at risk, and $34 \%$ of those who lacked a diagnosis but had experienced a fracture. According to $61 \%$ of the women surveyed who had discussed osteoporosis with their HCPs and who were at risk for osteoporosis, their HCPs had initiated conversations with them about osteoporosis; $49 \%$ of respondents not at risk affirmed that their HCPs had done this. Forty-one percent of respondents at risk for osteoporosis who had discussed osteoporosis with their HCPs and whose HCPs brought up osteoporosis answered that they thought the conversations with their HCPs about osteoporosis were started because of their age.

\section{Respondent views on the treatment of osteoporosis}

When asked about current and prior prescription medication for osteoporosis, $24 \%$ of women with self-reported osteoporosis reported prior but no current use of prescription osteoporosis medication, while $50 \%$ reported never receiving a prescription for osteoporosis treatment. Younger respondents were directionally more likely to be treatment naïve than older respondents (31\% of those aged $50-64$ years vs $19 \%$ of those aged 65 years and older) (Supplementary Table S1).

Twenty-seven percent of women with osteoporosis currently receiving a prescription medication had either taken a drug holiday or stopped their medication in the past. In $47 \%$ of cases, the respondents themselves decided on the holiday, and, in $48 \%$ of cases, their HCP recommended it.

Sixty-nine percent of women surveyed who were unaware that various treatment options are available for osteoporosis that build new bone or strengthen existing bone were willing to discuss these options with their physician. When women were asked about the type of medication they believed would be used first after an osteoporosis diagnosis, $88 \%$ of survey participants thought that first-line treatment of a fracture would be an agent designed to strengthen existing bone.

\section{Discussion}

This survey of US postmenopausal women with and without a self-reported osteoporosis diagnosis provides new insights regarding women's perceptions of this disease and its management. Based on their own self-reported risk factors, the 
Fracturing/breaking bones in a wrist or ankle after a clumsy trip/fall could happen to any woman my age

A fractured/broken bone for a woman my age could be life changing

When a fractured/broken bone heals a woman my age can get back to her normal life again

Eating healthy and exercising will prevent bone fractures/breaks

Fracturing/breaking one bone is a potential sign of more fractures/breaks to come

Only those that are more likely to have poor bone health are prone to fracturing/breaking bones

There is no way to build new bone at my age

The risk of a fracture/break is not able to be reduced for women my age

Only older (70+) women are likely to fracture/break their bones

There is not much I can do to prevent a fracture/break

$$
\begin{aligned}
& \text { Total, } \mathrm{n}=1012 \\
& \text { Dx with PMO, } \mathrm{n}=501
\end{aligned}
$$

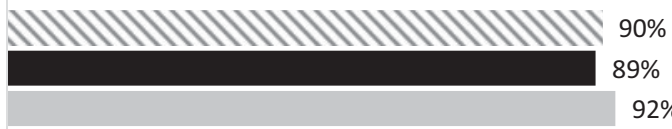

$92 \%$

$90 \%$ a
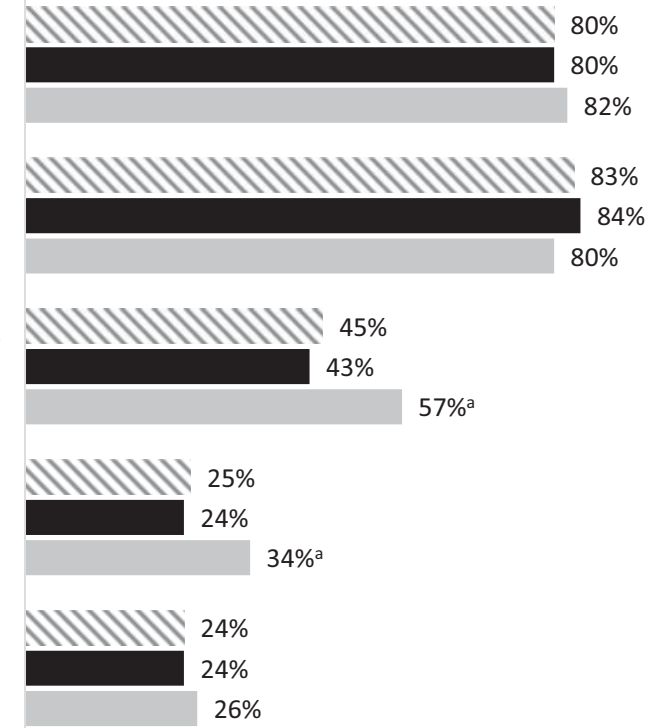

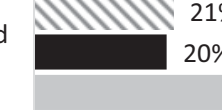

$21 \%$

$0 \%$

$30 \%$
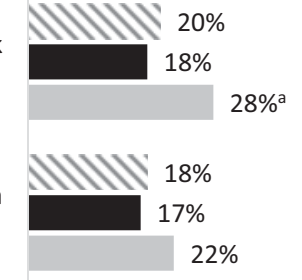

$\begin{array}{lllll}0 \% & 20 \% & 40 \% & 60 \% & 80 \%\end{array}$

- No PMO diagnosis, $\mathrm{n}=511$

Figure 3. Perceptions of the etiology and prognosis of osteoporosis by postmenopausal women. ${ }^{\mathrm{a}} p<0.05$. $t$-tests were conducted at the $95 \%$ confidence level $(\mathrm{CL})$, therefore representing a $p$-value $<0.05$.

majority of survey respondents (64\%) without an osteoporosis diagnosis had one or more risk factors for osteoporosis, consistent with the hypothesis that one in two Americans 50 years and older will have or be at high risk for osteoporosis by $2020^{2}$. Further, the finding that $34 \%$ of those with a fracture without an established diagnosis of osteoporosis did not recall having undergone diagnostic testing is consistent with the low rate of diagnostic testing reported by other investigators. The HEDIS Osteoporosis Measure assessment requires women aged 65-85 years who suffered a fracture be screened with a BMD test or prescribed osteoporosis treatment within 6 months following the fracture ${ }^{7}$. However, in a retrospective claims-based study of 1.6 million women without a history of an osteoporosis diagnosis or osteoporosis drug therapy (2008-2014), Gillespie and Morin ${ }^{10}$ found screening rates of only $21 \%$ for women aged 50-64 years, $27 \%$ for women aged $65-79$ years, and $13 \%$ for women 80 years and older. Only $24 \%$ of those with incident hip fractures were screened for osteoporosis following their fracture. In addition to the low diagnosis rate reported by our respondents, many women who had been diagnosed with osteoporosis under-estimated the significance of the condition and the consequences of sustaining a fracture. The survey further revealed patients' lack of awareness of poor bone health as a risk factor for fractures and the importance of a sentinel fracture as a consideration for an increased future fracture risk.

The current survey results suggest that at least one quarter of postmenopausal women believe that osteoporosis is associated only with hip and back fractures. This finding is 


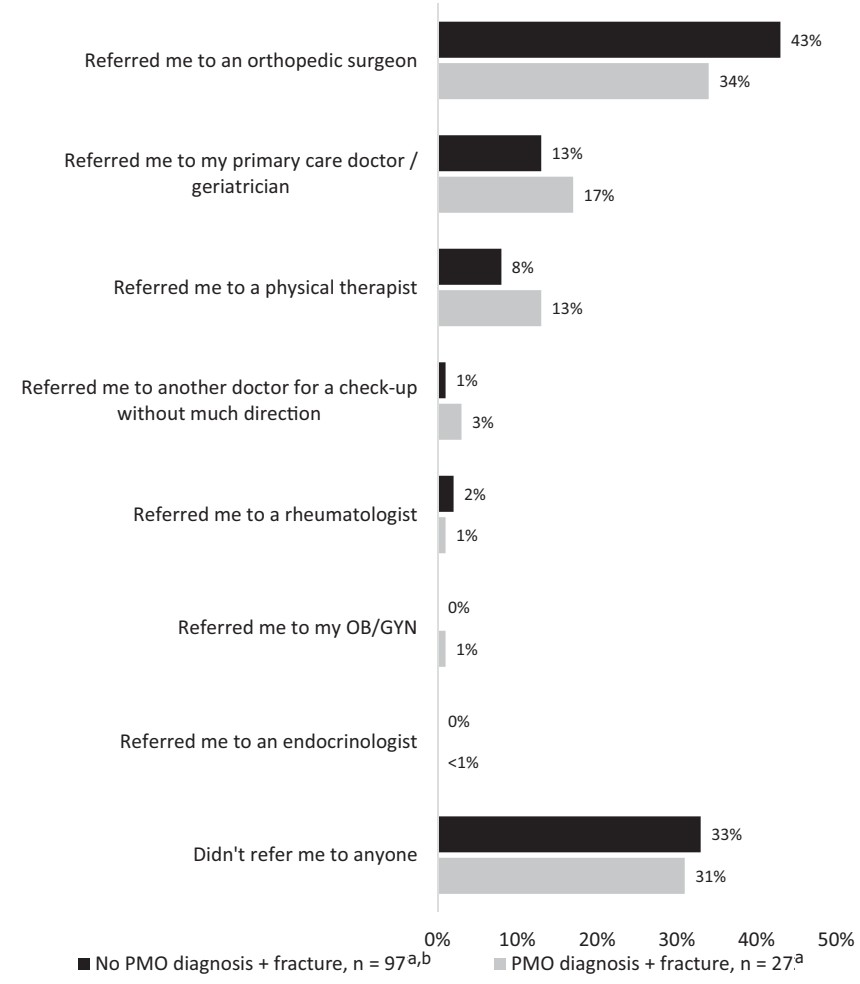

Figure 4. Referral for follow-up after a fracture from falling from a standing position or less. ${ }^{a}$ Respondents included here all saw an HCP after experiencing their fracture. ${ }^{b}$ Owing to sample size $<100$, these results are directional in nature.

troublesome, in that broad-based national surveillance programs estimate that between 2001 and 2007 more than 1 million American men and women 50 years and older were treated in hospital emergency departments for falls resulting in forearm or wrist fractures. After controlling for age, fracture rates are on average 3.1 times greater in women than in men. Eighty percent of fractures occurred in women, with rates increasing with age older than 50 years ${ }^{11}$. Moreover, a wrist fracture in women 65 years and older has been found to increase by $48 \%$ the odds of experiencing a clinically important functional decline over time ${ }^{12}$.

Misperceptions about the causes and consequences of fractures, coupled with poor guidance and missed opportunities for education from HCPs, are likely to contribute to a low likelihood of treatment initiation and adherence. Results from a recent survey of patients with osteoporosis and their physicians found that some patients may perceive osteoporosis severity differently from their physicians and have different concerns regarding the disease and its management ${ }^{13}$. Thus, improved communication between physicians and their patients regarding disease severity and future fracture risk may facilitate shared treatment decision-making. Although our survey did not include HCP perceptions, nor was there linkage to medical records to verify if communication was documented, respondents' recall of the absence of such communication is suggestive of a potential gap and an opportunity to address HCP perspectives in the future. Importantly, our survey suggests that HCPs may not effectively communicate to women the risk of having osteoporosis and its impact on future fractures.

\section{Limitations}

A key limitation of this survey is the generalizability of its findings to other individuals with, or at risk for, osteoporosis. Survey questions were directed at postmenopausal women, and responses to many questions were dependent on the age of the respondent. Additionally, physician perception, which may have provided more detailed insight into barriers associated with osteoporosis diagnosis and treatment, was not included in the survey. Another limitation is the inherent conflict between self-reported measures and what, in fact, occurred, including recall bias and lack of validation against medical records or physician perspectives. For example, among patients who lacked an osteoporosis diagnosis but had experienced a fracture, the survey did not make clear if screening was done prior to fracture occurrence or aftersomething that would likely have been gleaned from clinical records. Given the robust size of the survey population, however, we believe the likelihood of data distortion in our survey was small. Lastly, the survey was designed to be completed in 20 minutes, requiring us to restrict the number of ask-all and follow-up questions to a limited number of tightly focused topics.

\section{Conclusions}

These survey findings support the view that postmenopausal women could benefit from more education about osteoporosis. There is a need for increased awareness among women that a fracture is a sentinel event requiring evaluation and early intervention to reduce future fracture risk. Heightened disease awareness may enable patients to self-advocate to help ensure they are assessed for osteoporosis and, when appropriate, receive non-pharmacologic and pharmacologic therapy to reduce fracture risk.

On the basis of the current survey findings, we recommend that future research focuses on (1) validation of patient responses with medical records (for disease severity and risk factors); (2) evaluation of alignment between patient and physician perception of causes and management of fractures; (3) opportunities to improve communication regarding osteoporosis, fracture risk, and interventions to reduce fracture risk; and (4) exploration of a broader range of topics. Given the number of different specialties that treat osteoporosis, further education of HCPs regarding guidelines and treatment options for optimization of fracture risk management is warranted.

\section{Transparency}

\section{Declaration of funding}

This survey was conducted by The Harris Poll on behalf of Radius Health, Inc., Waltham, MA.

\section{Declaration of financial/other relationships}

EML has received consulting and/or speaker honoraria from Merck \& Co. AbbVie, AgNovos Healthcare, Alexion Pharmaceuticals, Amgen, Eli Lilly 
and Company, Radius Health, Inc., Shire, and TheraNova, along with research grant support from Merck \& Co., Amgen, and Eli Lilly and Company. He serves as a board member for the National Osteoporosis Foundation, the International Society for Clinical Densitometry, and the Osteoporosis Foundation of New Mexico. DL, SAW, and RW are employees of Radius Health. JDA peer reviewers on this manuscript have no relevant financial or other relationships to disclose.

\section{Acknowledgments}

The survey was conducted in partnership with HealthyWomen (www. healthywomen.org) and the National Osteoporosis Foundation (NOF; www.nof.org). Medical writing and editorial assistance was provided by Ian Eustace and Jason Scozzafava, Adelphi Communications, Bollington, UK and New York, NY, US; this assistance was funded by Radius Health, Inc.

\section{References}

[1] Burge R, Dawson-Hughes B, Solomon DH. Incidence and economic burden of osteoporosis-related fractures in the United States, 2005-2025. J Bone Miner Res. 2007;22:465-475.

[2] Bone health and osteoporosis: a report of the Surgeon General. Rockville, MD: US Department of Health and Human Services, Office of the Surgeon General, 2004; [cited 30 Jul 2018]. Available from: https://www.surgeongeneral.gov/library/.

[3] US Department of Commerce, Economics and Statistics Administration, US Census Bureau. The next four decades. The older population in the United States: 2010 to 2050. Population estimates and projections. Washington (DC): US Department of Commerce; [cited 30 Jul 2018]. Available from: https://www.cen sus.gov/prod/2010pubs/p25-1138.pdf.
[4] Amin S, Achenbach SJ, Atkinson EJ, et al. Trends in fracture incidence: a population-based study over 20 years. J Bone Miner Res. 2014;29:581-589.

[5] Leader D, Williams SA, Curtis JR, et al. Osteoporosis-related fracture events in 2015 in the US. Poster session presented at: AMCP Nexus; 2017 Oct 16-19; Dallas, TX.

[6] HEDIS ${ }^{\circledR}$ \& performance management, 2018. Washington (DC): National Committee for Quality Assurance; [cited 30 Jul 2018] Available from: http://www.ncqa.org/HEDISQualityMeasurement/ WhatisHEDIS.aspx.

[7] Osteoporosis testing and management in older women. Washington (DC): National Committee for Quality Assurance; [cited 30 Jul 2018]. Available from http://www.ncqa.org/report-cards/ health-plans/state-of-health-care-quality/2017-table-of-contents/ osteoporosis.

[8] Gillespie CW, Morin PE. Osteoporosis-related health services utilization following first hip fracture among a cohort of privatelyinsured women in the United States, 2008-2014: an observational study. J Bone Miner Res. 2017;32:1052-1061.

[9] Weaver JP, Olsson K, Sadasivan R, et al. Reasons for not treating women with postmenopausal osteoporosis with prescription medications: physicians' and patients' perspectives. J Women's Health. 2017;26:1302-1311.

[10] Gillespie CW, Morin PE. Trends and disparities in osteoporosis screening among women in the United States, 2008-2014. Am J Med. 2017;130:306-316.

[11] Orces C, Martinez FJ. Epidemiology of fall related forearm and wrist fractures among adults treated in US hospital emergency departments. Inj Prev. 2011;17:e33-e36.

[12] Edwards BJ, Song J, Dunlop DD, et al. Functional decline after incident wrist fractures-Study of Osteoporotic Fractures: prospective cohort study. BMJ. 2010;341:c3324.

[13] Gold D, Williams S, Weiss R, et al. Physician- and patient-reported disease severity in adults with osteoporosis: a US Cross-Sectional Survey. Endocrine Pract. 2018;24:Abst\#536. 